\title{
Photodetection of Low-Level Radiation through the Turbulent Atmosphere*
}

\author{
Paul Diament and M. C. Teich \\ Department of Electrical Engineering, Columbia University, New York, New York 10027
}

(Received 3 April 1970)

\begin{abstract}
Photoelectron-counting distributions are obtained for stochastic light that is caused to scintillate by passage through a random medium. The result is applied specifically to transmission of amplitude-stabilized radiation, with and without independent additive background, and of chaotic radiation, through the turbulent atmosphere. The counting distribution is found to broaden markedly and its peak occurs at decreasing count numbers for increasing turbulence. The cases studied here are of particular interest for low-level direct optical communications and radar using single-mode lasers, multimode lasers, thermal sources, and scattering targets. The results are obtained by extending the usual formulation of photoelectron counting; a product of two random variables, the source intensity and the effect of the turbulent medium, rather than the usual single stochastic irradiance, is considered. Plots of the counting distributions for various degrees of turbulence and for several signal-to-noise ratios are presented. A possible explanation is given for the observed decrease of the log-amplitude variance to values below the saturation value in long path length and high turbulence experiments.
\end{abstract}

Index Headings: Scintillation; Detection; Atmospheric optics.

The effects of the atmosphere on the irradiance fluctuations of a beam of radiant energy passing through it are important for optical communications and radar systems, and have been studied extensively. ${ }^{1-10}$ In all of the results discussed to date, however, the detected flux is relatively intense, and a continuous current is obtained from the photoelectric receiver. In this paper, we examine such systems at low irradiance levels and for short detection time intervals, whereupon the photodetector output consists of randomly distributed discrete pulses, rather than a continuous current. This work is therefore especially pertinent to laser communications and radar systems in which the path length is large and the bit rate for transmitting information is high.

Experimental work to date ${ }^{2-8}$ has been consistent with the prediction that the statistics of the irradiance fluctuations imposed by the atmosphere on otherwise steady radiation are log normal; i.e., the logarithm of the irradiance, and also of the field amplitude, is normally distributed. A simple model for this result envisions it as arising from the cumulative action of successive refractive-index irregularities, or packets of turbulence, encountered by the beam. ${ }^{1,3}$ The effect of each of these on the flux reaching the detector is representable by a random multiplicative factor. The logarithm of the radiant power, after transmission through the atmosphere, is then a sum of that of the source and a large number of additive, independent, stochastic variables. By the central-limit theorem, this sum of logarithms ought to be normally distributed, with a variance related to the level of atmospheric turbulence. ${ }^{1-10}$

In the experiments reported so far, the bandwidth of the photodetector is sufficiently large (usually of the order of kilohertz) for the photodetector current to follow the time development of the log-normally distributed stochastic irradiance at the detector. The photocurrent itself is then also log-normally distributed and it is this continuous current that has been studied. Stochastic fluctuations of the intensity of the source may also occur, however, and usually at a rate more 
rapid than that of the atmospheric fluctuations. In conventional experiments, these fluctuations fall outside the photodetector bandwidth and are therefore not seen. By contrast, in counting experiments, the observation time $T$ may be smaller than the coherence time $\tau_{\theta}$ of the source radiation, in which case the resultant counting distribution will depend not only on the atmospheric fluctuations but on the source radiation statistics as well.

We inquire here into the photoelectron-counting statistics to be expected from stochastic radiation, after it has passed through the turbulent atmosphere. Counting-time intervals $T$ that are short compared to both the atmospheric fluctuation time $\tau_{a}$ and the source coherence time $\tau_{c}$ are considered, as well as several other combinations of time scales. The photoelectric detector is taken to be signal-noise as opposed to thermal-noise limited ${ }^{11}$ and, for the visible region of the spectrum, is grenerally a photomultiplier. We specifically investigate the cases of a stable ideal single-mode laser source, a chaotic source, and a stable source plus independent additive noise, such as stray light or detector dark current.

\section{COUNTING STATISTICS}

The photoelectron-counting statistics for fluctuating light passing through a medium whase transmittance varies stochastically may be obtained as an extension of Mandel's formula. ${ }^{12-18}$ Although the counting distribution may still be expressed as

$$
p(n)=\left\langle\left(E^{n} / n !\right) e^{-E}\right\rangle
$$

the stochastic variable

$$
E=\int_{t}^{t+T} \alpha\left(t^{\prime}\right) I\left(t^{\prime}\right) d t^{\prime}
$$

must be reexamined. In the usual formulation, this is the exposure of the photodetector during a time $T$ to an irradiance $I$, with $\alpha$ a constant incorporating the quantum efficiency and the area of the photodetector. For $T \ll \tau_{c}$, averaging with respect to $E$ has been considered equivalent to averaging with respect to the statistics of the source intensity. In the present case, the irradiance fluctuations are a resultant of those of the source intensity and those imposed by the turbulent medium. In many cases, both effects can be accounted for while leaving Eq. (2) intact if $I$ is reinterpreted as the source intensity and $\alpha$ is taken to include not merely the quantum efficiency of the detector but also the overall transfer function from source to photosensitive surface. This can include the effects of various optical components, as well as the characteristics of the media through which the light has passed. The factor $\alpha$ can hence be time varying, either deterministically or stochastically, and must then remain under the integral in Eq. (2). This model is valid when the medium between the source and the detector acts as a linear irradiance filter or, as in the present case, when an effective multiplicative function, which may result from interference, can be found. To determine the statistics of the exposure $E$, it is assumed that fluctuations in $I$ and in $\alpha$ are statistically independent.

Actually, any or all of the quantities $I, \alpha, b$, and $T$ that determine the exposure in $\mathbf{E q}$. (2) may be random. Various investigators have implicitly considered several of these as time varying, but generally only one as random. We consider both $\alpha$ and $I$ to be randomly fluctuating, independently, with both processes stationary, so that the results are independent of $t$ in Eq. (2). We emphasize that when modulation of the type specified above is introduced between the source and the detector, it is $\alpha$ rather than $I$ that is to be considered as varied in time, although the distinction is unimportant until both $\alpha$ and $I$ vary, independently. For $T \ll \tau_{c}, \tau_{a}$, such variations imposed on the instantaneous irradiance are equivalent to modulation of its mean, as we indicated in earlier work dealing with square-wave, sinusoidal, and triangular modulation of a stochastically radiating source. ${ }^{18}$

\section{ANALYTICAL RESULTS}

Consider quasimonochromatic light directly incident on a photoelectron counter, yielding an average of $N$ counts in an observation-time interval $T$. This count rate is proportional to the mean irradiance. The actual count in a particular observation is random, with a discrete probability distribution $p_{0}(n, N)$. This is conventional photoelectron counting notation, which might be more properly written as $p_{0}(n / N)$. For example, amplitude-stabilized radiation leads to a Poisson distribution of the photoelectrons ${ }^{12-18}$

$$
p_{0}(n, N)=\left(N^{n} / n !\right) e^{-N} .
$$

Chaotic radiation, as from a thermal source, gives rise to photoelectron counts with a Bose-Einstein, or geometric distribution ${ }^{12-18}$

$$
p_{0}(n, N)=N^{n} /(N+1)^{n+1},
$$

provided that $T \ll \tau_{c}$ and the detector area is much smaller than the coherence area.

If this light now passes through the atmosphere, a random modulation of the mean irradiance is imposed on it. The corresponding modulated mean count $K$ during an observation period $T$ then has the (continuous) $\log$-normal distribution

$$
P(K)=\exp \left(-\left[\ln (K / N)+\frac{1}{2} \sigma^{2}\right]^{2} / 2 \sigma^{2}\right) /(2 \pi)^{\frac{1}{2}} \sigma K,
$$

where $N$ is the over-all mean of the counts produced by the doubly fluctuating light at the detector and $\sigma$ is the standard deviation of the logarithm of $K$.

The photoelectron counting statistics for the radiation modulated by the atmosphere are given $b y^{18}$ 


$$
p(n, \sigma, N)=\int_{0}^{\infty} p_{0}(n, K) P(K) d K,
$$

where $p_{0}(n, K)$ is the distribution of counts, with mean $K$, that would be obtained in the absence of the atmosphere. The mean count $N$ and the logarithmic standard deviation $\sigma$ are parameters in $P(K)$. We assume that the modulation imposed by the atmosphere does not affect the radiation statistics of the source, so that the two random processes are independent. This also justifies considering the modulation as imposed on the mean irradiance, rather than on the instantaneous value.

A general evaluation of the integral in Eq. (6) may be made by the method of steepest descent. The variable of integration is changed to $\ln K$ and the undisturbed probability distribution $p_{0}(n, K)$ is expressed as an exponential of its own logarithm. The point of stationarity for the combined exponential is then sought and a Taylor-series expansion about that point readily yields an approximate expression for the modulated-light counting statistics. The approximation is excellent under weak conditions on the form of the unmodulated distribution $p_{0}(n, K)$.

Formally, let

$$
q_{m}(n, N)=\partial^{m} \ln p_{0}(n, N) / \partial(\ln N)^{m}
$$

and determine $M$ implicitly for each $n$ from the stationarity condition

$$
\ln M=\ln N-\frac{1}{2} \sigma^{2}+\sigma^{2} q_{1}(n, M) .
$$

A power-series expansion in the variable

$$
x=[\ln (K / M)] / \sigma
$$

is to be made. Thus, in the integral,

$$
P(K) d K=\exp \left[-\frac{1}{2}\left(x+\sigma q_{1}\right)^{2}\right] d x /(2 \pi)^{\frac{1}{2}}
$$

and

$$
p_{0}(n, K)=p_{0}(n, M) \exp \left(x \sigma q_{1}+\frac{1}{2} x^{2} \sigma^{2} q_{2}\right) \exp R(x),
$$

in which the remainder term $R(x)$ is of order $x^{3} \sigma^{3} q_{3}$. Provided that this can be neglected in comparison with $x^{2}\left(1-\sigma^{2} q_{2}\right)$ in the exponent, the integral in Eq. (6) yields the approximation

$$
p(n, \sigma, N)=\frac{p_{0}(n, M) \exp \left[-\frac{1}{2} \sigma^{2} q_{1}^{2}(n, M)\right]}{\left[1-\sigma^{2} q_{2}(n, M)\right]^{\frac{1}{2}}} .
$$

The result applies to any reasonable, single-peaked distribution $p_{0}(n, N)$.

For the case of amplitude-stabilized radiation, the Poisson distribution given by Eq. (3) yields

$$
q_{1}(n, N)=n-N, \quad q_{2}(n, N)=-N,
$$

so that the procedure requires the solution of

$$
\ln M=\ln N+\sigma^{2}\left(n-\frac{1}{2}-M\right)
$$

for $M(n)$, with $N$ and $\sigma$ as parameters, for each $n$. Then

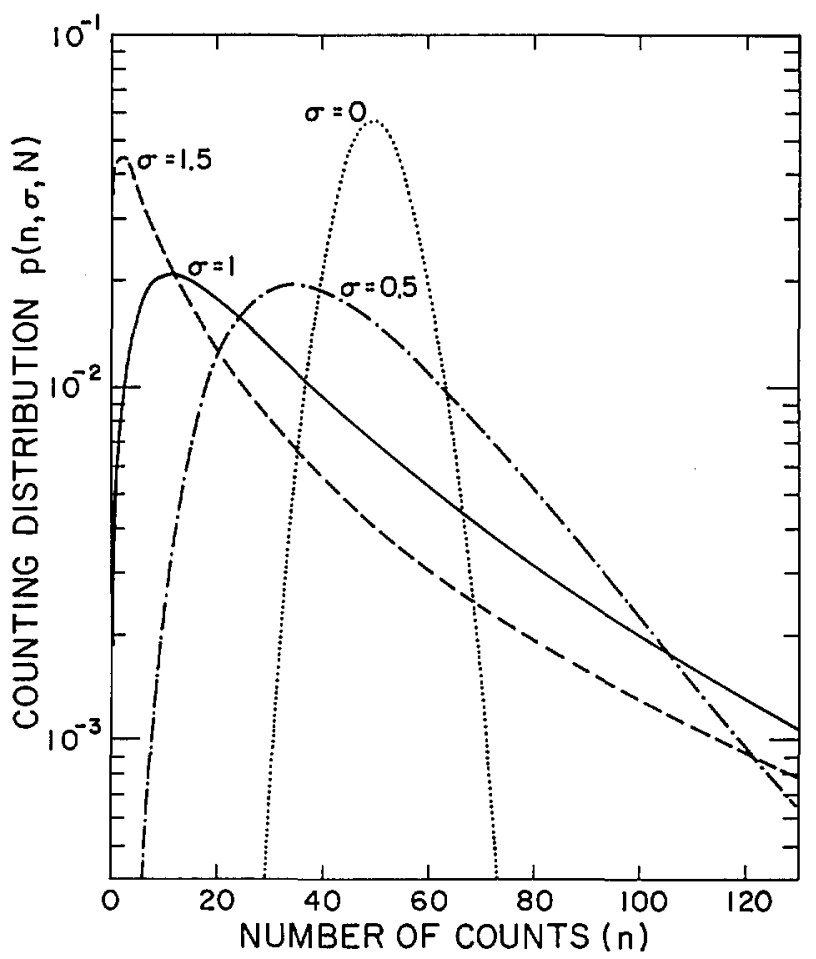

FIG. 1. Photoelectron counting distributions for amplitudestabilized light, after transmission through the atmosphere. The level of turbulence is indicated by the logarithmic-irradiance standard deviation $\sigma$. The mean count is $N=50$ for all cases shown.

the photoelectron-counting statistics for amplitudestabilized light that has passed through the atmosphere are given very closely by

$$
p(n, \sigma, N)=\frac{M^{n} e^{-M} \exp \left[-\frac{1}{2} \sigma^{2}(M-n)^{2}\right]}{n !\left[1+\sigma^{2} M\right]^{\frac{1}{2}}} .
$$

Figure 1 presents plots of this distribution for a mean count of $N=50$, with various values of the log-intensity standard deviation $\sigma$. These results should describe the photodetection statistics for single-mode-laser light passing through the atmosphere.

For the case of chaotic radiation, the geometric distribution of the unmodulated light given in Eq. (4) yields

$$
\begin{gathered}
q_{1}(n, N)=(n-N) /(N+1), \\
q_{2}(n, N)=-(n+1) N /(N+1)^{2} .
\end{gathered}
$$

For each $n$, the equation to be solved for $M$ is

$$
\ln M=\ln N-\frac{1}{2} \sigma^{2}+\sigma^{2}(n-M) /(M+1)
$$

and the corresponding counting distribution for chaotic light passing through the atmosphere is approximately

$$
\begin{aligned}
& p(n, \sigma, N) \\
& \quad=\frac{[M /(M+1)]^{n} \exp \left(-\frac{1}{2} \sigma^{2}[(M-n) /(M+1)]^{2}\right)}{\left[(M+1)^{2}+\sigma^{2}(n+1) M\right]^{\frac{1}{2}}} .
\end{aligned}
$$




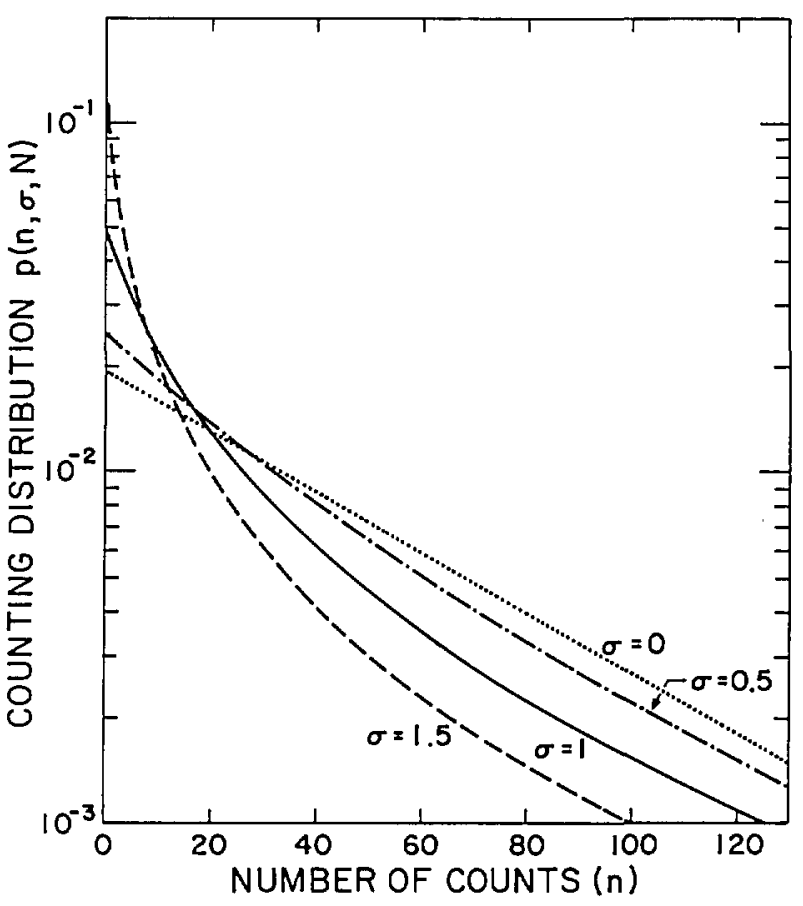

FIG. 2. Photoelectron-counting distributions for light from a chaotic source after transmission through the atmosphere. The level of turbulence is indicated by the logarithmic-irradiance standard deviation $\sigma$. The mean count is $N=50$ for all cases shown.

Plots corresponding to those of Fig. 1, but for chaotic radiation, are presented in Fig. 2.

\section{DISCUSSION OF NOISELESS CASE}

The over-all mean of the counting distributions shown in Figs. 1 and 2 has been arbitrarily chosen to be $N=50$; the general shape and behavior of the curves is relatively independent of $N$. The data have been given for values of the log-intensity standard deviation of $\sigma=0,0.5,1,1.5$. The limit $\sigma=0$ corresponds to a quiescent atmosphere, for which all atmospherically imposed fluctuations disappear and the counting distribution reverts to the undisturbed one. In Fig. 1, this is the usual Poisson distribution that arises from a stable source. As the strength of the atmospheric fluctuations increases, the counting distribution gradually broadens and the peak of the distribution shifts to a decreasing count number $n$. For $N=50$, the peak occurs at $n=50$, 34,11 , and 2 for $\sigma=0,0.5,1,1.5$, respectively. At somewhat higher values of $\sigma$, the most likely count becomes $n=0$. The highest value indicated, $\sigma=1.5$, corresponds to a strongly turbulent atmosphere. To make connection with experimental studies, ${ }^{2-8}$ we note that $\sigma^{2}=4 C_{l}(0)$, where $C_{l}(\rho)$ is the log-amplitude two-point covariance function; $C_{l}(0)$ is the generally reported variance. The dependence of $C_{l}(0)$ on atmospheric turbulence and on the parameters of a particular system has been the subject of much debate and discussion. ${ }^{1-10}$ It seems clear from experiments. however. that this variance saturates with increasing atmospheric turbulence and is generally restricted to $0 \lesssim C_{l}(0) \lesssim 0.6$, corresponding to $0 \lesssim \sigma \lesssim 1.5$. The apparent value of $\sigma$ in an experiment also depends on the detector area; it has been shown by Fried et al. ${ }^{2,}$ to be a decreasing function of receiver area. Under such aperture averaging, the statistics for the amplitude-stabilized source appear to remain log normal. However, in a different model for the atmosphere, in which Rayleigh amplitude fluctuations are imposed on amplitude-stabilized light traversing it, ${ }^{9}$ the irradiance distribution becomes Rice-Nakagami rather than log normal. The corresponding photoelectron-counting distribution has been considered previously in connection with laser studies, ${ }^{13,14,18}$ although it has not been applied to photodetection through the atmosphere. The fluctuations are less severe and the resultant photoelectron-counting distributions less broad and more concave downward than for the amplitudestabilized log-normal case. Details of these calculations may be obtained in the literature and are not discussed here.

The results for the chaotic source are given in Fig. 2 . They apply to thermal sources, free-running multimode lasers, and single-mode laser light scattered from a rough target, ${ }^{19}$ as in a laser radar, for sufficien tly small detector resolution time and area. For $\sigma=0$, viz., no log-normal atmospheric scintillation, the counting statistics revert to the Bose-Einstein distribution, a straight line in this semilogarithmic plot. As $\sigma$ increases, the curvature of the counting distribution becomes greater, thus emphasizing low and high counts at the expense of counts near the mean. This is a result of the tendency of atmospheric fluctuations to cause clusters of photons, few arriving during times of destructive interference and many during times of constructive interference. An analogous effect occurs for deterministic modulation, where increasing modulation depth causes the curvature to increase. ${ }^{18}$ Comparing the chaotic and stable sources, the effect of the atmosphere on the counting distribution is smaller for the chaotic source, which has large preexisting fluctuations, and therefore a broad counting distribution, even in the absence of modulation. The otherwise narrow, peaked Poisson distribution for the stable source is altered considerably by the turbulence. In fact, for high values of $\sigma$, the counting distribution becomes relatively insensitive to the source statistics and is determined primarily by those of the atmospheric scintillations.

The preceding discussion is applicable for short counting intervals, $T \ll \tau_{c}, \tau_{a}$, and for a receiver area smaller than the coherence area. As discussed in an earlier paper, ${ }^{18}$ for $\tau_{c} \ll T \ll \tau_{a}$, fluctuations due to the source are averaged out and the results are identical to those given by Eqs. (14) and (15) for the stable source. This is probably the condition most likely to be satisfied in practice. In the opposite limit, $\tau_{a} \ll T \ll \tau_{c}$, only fluctuations due to the source are observed and atmospheric modulation need not be considered. Similarly, for 
$T \gg \tau_{c}, \tau_{a}$, no fluctuations are resolved by the detector, and a simple Poisson distribution results. The counting distribution for atmospherically disturbed chaotic light with arbitrary $\tau_{c} / T$ (but still subject to $T \ll \tau_{a}$ or $T \gg \tau_{a}$ ) could be obtained by using the approximate distribution of Bédard, Chang, and Mandel ${ }^{20}$ for this case. In the usual experimental situation, $\tau_{a}$ is of the order of milliseconds and thus, for a reasonable bit rate, $T \ll \tau_{a}$, which is the case to which we have primarily addressed ourselves.

\section{DETECTION OF LIGHT WITH ADDITIVE NOISE}

Consider noise, in the form of background light, entering the detector from sources independent of the transmitter. We assume that the signal and noise beams do not interfere and that their irradiances are therefore additive. Under usual conditions, the ambient light has a relatively large bandwidth, even as seen through a narrow interference filter $(\gtrsim 1 \AA)$, and therefore has a very short noise coherence time $\tau_{n}$. If we assume that $T \gg \tau_{n}$, then the rapid fluctuations of the noise will not be seen. This sort of background light will therefore lead to a Poisson distribution of photoelectron counts in the absence of signal light. Furthermore, dark current is also manifested by a Poisson distribution and may be combined with that of the ambient light. The superposition of the additive signal and noise contributions vields a counting distribution expressible as the convolution of the individual counting statistics, $p_{S}$ and $p_{N}$,

$$
\begin{aligned}
& p_{S+N}(n, \sigma, N) \\
& \quad=\sum_{m=0}^{n} p_{S}[m, \sigma, N y /(1+y)] p_{N}[n-m, N /(1+y)],
\end{aligned}
$$

where $y$ is the signal-to-noise ratio (SNR). This result may also be obtained by viewing the additive noise as the minimum effective exposure in Mandel's formula ${ }^{12}$; i.e., shifting the probability density of the signal exposure $E$ by the average level of this noise.

- Figure 3 presents a plot of Eq. (20) for the particular case of Poisson noise and a signal-counting distribution obtained from a stable source whose light passes through a moderately turbulent atmosphere, with $\sigma=1$. The mean count remains the same, at $N=20$, whereas the relative contributions arising from signal and noise change with the indicated SNR. For $y=0$, the distribution is simply Poisson with a mean count of 20 ; in this case all of the counts arise from noise. In the opposite limit, for $y=\infty$, the distribution is that obtained in the noiseless case and is identical to that obtained from Eqs. (14) and (15). As the SNR increases from zero, the distribution gradually broadens, while its peak shifts toward lower count number. The shape of the curve for $y=4$ is not very different from that at $y=\infty$, indicating that the detection process becomes relatively insensitive to noise at even moderate SNR's.

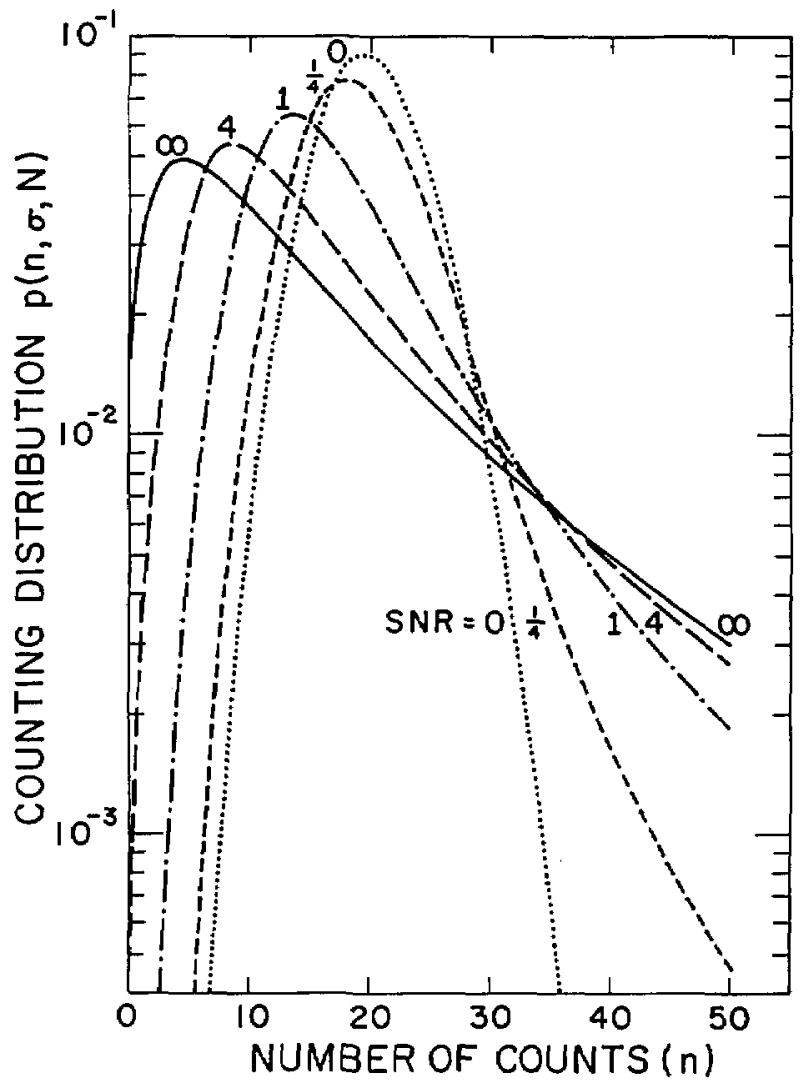

FIG. 3. Photoelectron-counting distributions for combinations of amplitude-stabilized light after passage through a turbulent atmosphere $(\sigma=1)$ with independent, noninterfering, Poissondistributed noise counts. The combined mean count is $N=20$ for each SNR.

\section{CONCLUSION}

Light transmitted through the turbulent atmosphere undergoes irradiance fluctuations that result in an altered photoelectron-counting distribution after photodetection. The usual treatment of photoelectron counting has been extended so that an intervening medium such as the atmosphere, as well as the source intensity, may be stochastic. When the two processes are independent, it is sufficient to consider the log-normal statistics of the atmosphere as modulating the mean count of the undisturbed distribution. The calculated counting distributions for an atmospherically distorted amplitude-stabilized source, with and without independent, noninterfering background radiation, and for a chaotic source, reveal the characteristic increased likelihood of lower counts. In predicting these distributions, we have assumed that the log-normal statistics of the continuous photocurrent that has been observed in various experiments are a sufficiently complete description of the stochastic effect of the atmosphere. If, for example, additional fast irradiance fluctuations were imposed by the atmosphere, these might be evident in fast photoelectron-counting receivers, and not in the 
slower continuous-current experiments performed so far. Thus, experimental verification of the results presented in this paper would not only validate the assumed log-normal statistics, but might provide new information.

To obtain the counting distributions, the averaging integral in Eq. (6) has been evaluated by the saddlepoint method, involving the logarithmic derivatives of the logarithm of the undisturbed counting distribution. This yields reliable results whenever that distribution has a well-defined peak. Clearly, the method must be applied with appropriate care if multiple peaks in the unperturbed counting distribution can yield more than one stationary point in the integration. This can occur when the source radiation is modulated. ${ }^{18}$

We have specifically discussed the situation in which background light entering the detector is independent of, and does not interfere with, the signal light. Such ambient light, along with detector dark current, will usually give rise to a Poisson distribution of counts in the absence of signal. More general situations can be treated in a similar manner, however. If the counting statistics for the background light are other than Poisson, but still independent of the signal beam and noninterfering, Eq. (20) still applies, but with the appropriate noise-counting distribution $p_{N}$. If the background light interferes with the atmospherically distorted signal wave, and the beats fall within the detector bandwidth, then the irradiance statistics at the detector must be determined by adding electric fields rather than irradiances. When the appropriate irradiance distribution for the combined background and atmospherically distorted signal light is found, the usual Mandel formula ${ }^{12}$ may be applied. Other sources of noise, additive, independent, and noninterfering, may then be accounted for by a convolution, as in Eq. (20). Several recent treatments of cases involving interference terms have appeared ${ }^{16,17}$; the effects of log-normal atmospheric fluctuations on such radiation (e.g., from a non-ideal or laboratory laser) are presently being investigated.

For experiments over large distances, and for extremely high turbulence levels, the SNR $y$ is likely to decrease because of increased background and stray light entering the detector. A comparison of Fig. 3 with Fig. 1 shows that the effect of decreasing the $S N R$ is similar to that of decreasing the log-irradiance standard deviation $\sigma$. Thus, recent evidence that $\sigma$ appears to drop below its saturation level for high turbulence and long-path-length experiments $s^{5,6}$ may be more indicative of an increased fraction of stray light entering the detector than of $\sigma$ decreasing below its saturation value. In addition, the statistics would depart from log normal under these conditions, possibly contributing to experimentally observed deviations.

The ability to predict photoelectron-counting statistics for radiant energy of general time variation passing through the turbulent atmosphere extends the feasibility of laser communications and radar to the regime of low power levels or high sampling speeds, and may suggest modulation schemes that could diminish some of the effects of atmospheric turbulence.

\section{REFERENCES}

* This work was supported in part by the National Science Foundation under Grant NSF-GK-16649.

${ }^{1}$ V. I. Tatarski, Wave Propagation in a Turbulent Medium (McGraw-Hill, New York, 1961).

2 D. L. Fried, J. Opt. Soc. Am. 57, 169 (1967).

${ }^{3}$ D. L. Fried, G. E. Mevers, and M. P. Keister, Jr., J. Opt. Soc. Am. 57, 787 (1967).

4 D. L. Fried and R. A. Schmeltzer, Appl. Opt. 6, 1729 (1967).

${ }^{5}$ G. R. Ochs and R. S. Lawrence, J. Opt. Soc. Am. 59, 226 (1969).

${ }^{6}$ G. R. Ochs, R. R. Bergman, and J. R. Snyder, J. Opt. Soc. Am. 59, 231 (1969).

${ }^{7}$ P. H. Deitz and N. J. Wright, J. Opt. Soc. Am. 59, 527 (1969).

${ }^{8}$ G. E. Mevers, M. P. Keister, Jr., and D. L. Fried, J. Opt. Soc. Am. 59, 491A (1969).

${ }^{9}$ D. A. deWolf, J. Opt. Soc. Am. 59, 1455 (1969)

${ }^{10} \mathrm{~J}$. W. Strohbehn, Proc. IEEE 56, 1301 (1968).

${ }^{11}$ W. K. Pratt, Laser Conmunications Sysiems (Wiley, New York, 1969), p. 145.

${ }^{12}$ L. Mandel, Proc. Phys. Soc. (London) 72, 1037 (1958).

${ }^{13} \mathrm{~J}$. A. Armstrong and A. W. Smith, in Progress in Optics VI, edited by E. Wolf (North-Holland, Amsterdam, 1967), p. 213

${ }_{14}$ M. C. Teich and P. Diament, J. Appl. Phys. 40, 625 (1969); Phys. Letters 30A, 93 (1969); J. Appl. Phys. 41, 415 (1970).

${ }_{15}$ R. F. Chang, V. Korenman, C. O. Alley, and R. W. Detenbeck, Phys. Rev. 178, 612 (1969).

${ }_{10}$ E. Jakeman and E. R. Pike, J. Phys. A2, Ser. 2, 115 (1969)

17 J. Peřina and R. Horák, J. Phys. A2, Ser. 2, 702 (1969).

18 P. Diament and M. C. Teich, J. Opt. Soc. Am. 60, 682 (1970).

${ }^{19}$ M. C. Teich, in Semicondactors and Semimetals, edited by R. K. Willardson and A. C. Beer (Academic, New York, 1970), Vol. 5 (Infrared Detectors), p. 361. See also Proc. IEEE 57, 786 (1969) and Appl. Phys. Letters 14, 201 (1969).

${ }_{20}$ G. Bédard, J. C. Chang, and L. Mandel, Phys. Rev. 160, 1496 (1967). 